01

\title{
Возможности формулы Морза при аппроксимации энергии колебательных уровней двухатомной молекулы
}

\author{
(C) Г.С. Денисов, К.Г. Тохадзе \\ Санкт-Петербургский государственный университет, \\ 199034 Санкт-Петербург, Россия \\ e-mail: gldenisov@yandex.ru, kgtokhadze@yandex.ru \\ Поступила в редакцию 26.06.2021 г. \\ В окончательной редакции 26.06.2021 г. \\ Принята к публикации 13.07.2021 г.
}

\begin{abstract}
Рассмотрена задача аппроксимации потенциала двухатомной молекулы модельной функцией Морза с постоянной ангармоничностью $v x$ при использовании экстраполяции Берджа-Шпонер. Анализ приближений, использованных при выводе уравнения Морза, показывает, что решение этой задачи неоднозначно. Предложена схема оптимизации выбора исходных параметров, которая иллюстрирована примерами, взятыми из литературы. Продемонстрирована польза изучения поведения ангармоничности при возбуждении колебательных уровней по отклонениям величины $v x$ от постоянного значения по Морзу. Предпринята попытка использовать безразмерный параметр ангармоничности $x^{*}$ в качестве универсальной характеристики особенностей формы электронного терма молекулы.
\end{abstract}

Ключевые слова: формула Морза, двухатомная молекула, экстраполяция Берджа-Шпонер, ангармоничность, электронные термы, колебательная структура.

DOI: $10.21883 / \mathrm{OS} .2021 .11 .51635 .2483-21$

В курсах молекулярной спектроскопии модельный потенциал Морза традиционно упоминается всегда при изложении принципов формирования колебательной структуры спектров простых молекул и методов приближенной оценки энергии химической связи. Несмотря на весьма грубое приближение и часто лишь полуколичественное согласие с экспериментом, эта функция сохраняется в учебных планах и в учебных пособиях вплоть до последнего времени [1-7], а в научной литературе развиваются многообразные ее применения и модификации [8-12]. Основной причиной не снижающейся популярности функции Морза является интегрируемость уравнения Шредингера с таким потенциалом и богатство задач, в которых высока потребность в приблизительном оценочном решении, позволяющем осуществлять дальнейшую нацеленную проработку. Благодаря простоте и наглядности функция Морза легко усваивается студентами, но одновременно в образовательной системе формируются шаблоны, тормозящие эволюционное развитие. Остановимся на важной детали, которая не была до конца осознана в литературе за почти сто лет применения этого потенциала - о возможности вариации параметров функции при аппроксимации реальных термов и на развитии методологии описания ангармоничности.

\section{Альтернативные аппроксимации реального электронного терма}

Для аппроксимации реального электронного терма уравнением Морза [13]

$$
U(r)=D_{e}\left[1-e^{-a\left(r-r_{e}\right)}\right]^{2}
$$

необходимо задаться тремя значениями параметров $D_{e}$, $r_{e}$ и „a“. Смысл $D_{e}$ (глубина потенциальной ямы) и $r_{e}$ (положение минимума потенциальной кривой) ясен, а постоянная „,a“, определяющая ширину потенциальной ямы и само существование диссоциационного предела, входит в коэффициенты решения уравнения Шредингера с этим потенциалом:

$$
G(v)=v_{e}\left(v+\frac{1}{2}\right)-v_{e} x_{e}\left(v+\frac{1}{2}\right)^{2},
$$

где

$$
v_{e}=a\left(2 D_{e} / M \hbar\right)^{1 / 2}, \quad v_{e} x_{e}=\hbar a^{2} / 2 M,
$$

$v_{e}$ - гармоническая частота, $M$ - приведенная масса молекулы, $v_{e} x_{e}-$ коэффициент ангармоничности. Отсюда $a=2 \pi v_{e}\left(M / 2 D_{e}\right)^{1 / 2}$. Из (3) приближенно следует, что

$$
D_{e}=v_{e}^{2} / 4 v_{e} x_{e} .
$$

Это последнее соотношение позволяет использовать для определения , ,a“ вместо $D_{e}$ ангармоничность $v_{e} x_{e}$, или только $x_{e}$, и именно этот вариант предлагает Морз в качестве окончательного результата своей работы (статья [13] называется „Diatomic molecules according to the wave mechanics. II. Vibrational levels.“') и использует его для определения энергии диссоциации ряда двухатомных молекул путем экстраполяции. В дальнейшем при использовании уравнения Морза (1) авторы чаще всего указывают первый вариант. Он также чаще приводится в учебниках и монографиях [2-7]. Иногда авторы приводят оба варианта, упоминая об их эквивалентности, например в [14,15], хотя выражение (4) отягощено систематической ошибкой, природа которой заслуживает 
упоминания, поскольку она неизбежно ведет к двум возможным значениям параметра , $a^{6 .}$.

Первая систематическая ошибка заложена в дефиниции глубины потенциальной ямы $D_{e}$, которую Морз принимает равной положению последнего колебательного уровня $v_{\max }$, и таким образом, величина $D_{e}$ оказывается заниженной на величину, несколько меньшую последнего колебательного кванта. Из (2) формально следует

$$
v_{\max }=v_{e} / 2 v_{e} x_{e}-1 / 2
$$

но при использовании целочисленной части вносится вторая систематическая ошибка. Данные соображения в литературе в явном виде не упоминаются, хотя отсюда следует, что параметр „a“, выраженный через ангармоничность $v_{e} x_{e}$ по (4), занижен за счет систематической ошибки (5). Это ведет к завышению величины $D_{e}$ и, возможно, к завышению числа колебательных уровней.

В качестве конкретного примера мы провели аппроксимацию потенциальной кривой с колебательной структурой для молекулы водорода в основном электронном состоянии, использовав два набора параметров, включающих либо $D_{e}$, либо $x_{e}$, с использованием литературных данных. В первом случае получилась картина, похожая на схему рис. 48 в книге [7], с той разницей, что реальные колебательные уровни в верхней части потенциальной ямы сбегаются гораздо быстрее, чем вытекает из условия постоянства ангармоничности, число уровней возросло с 14 до 16. Это естественно, поскольку в обоих случаях аппроксимация мало эффективна в верхней части потенциала, выше $30000 \mathrm{~cm}^{-1}$ (примерно $20 \%$ от $D_{e}$ ), из-за различной асимптотики реальной и морзовской кривых. Во втором случае исходное значение ангармоничности $x_{e}$, вычисленное по экспериментальным значениям первых колебательных частот, уменьшилось, и в результате положение колебательных уровней было воспроизведено лучше в нижней части ямы почти до $30000 \mathrm{~cm}^{-1}$. Но положение асимптоты возросло больше, чем на $3000 \mathrm{~cm}^{-1}$, и число колебательных уровней увеличилось до 18. Отсюда следует, что исходный набор уровней, включающий ангармоничность, предпочтителен в случаях, когда важна воспроизводимость в нижней части потенциальной ямы, но энергия диссоциации при этом экстраполируется очень грубо. Если же энергия диссоциации известна, аппроксимация первого типа позволяет получить значения частот и волновые функции состояний в более широком интервале, но с несколько меньшей точностью. Добавим, что именно этот подход давно и широко используется при теоретическом изучении динамического взаимодействия сильного лазерного излучения с веществом, когда происходит накачка по колебательным степеням свободы молекулы вплоть до диссоциации и перехода в сплошной спектр [16-18]. В этих случаях более важно точнее знать энергию диссоциации, чем закон упорядоченности собственных частот ангармонического осциллятора.
Для вычисления коэффициента ангармоничности $x_{e}$ достаточно знать положение первых трех колебательных уровней реальной молекулы, но если известно их большее число, появляются новые возможности. Система уравнений, используемых для выбора значения $x_{e}$ (и одновременно связанного с ним значения $v_{e}$ ), становится избыточной, и оптимизация решения может быть обусловлена спецификой задачи. Во-первых, усредняя данные по частотам всех известных состояний, можно получить морзовскую аппроксимацию реальной потенциальной кривой на широком возможном интервале энергии, хотя и с неизбежной потерей точности для высоких уровней. Во-вторых, для некоторых задач может возникнуть потребность оптимального описания некоторой группы возбужденных колебательных состояний, не включающей одно или несколько самых нижних, и тогда коэффициент ангармоничности целесообразно определять усреднением данных по частотам именно этих состояний. В-третьих, опыт показывает, что нередко нижние уровни ведут себя аномально, т.е. сбегаются медленнее, чем вышележащие, и это приводит к неправдоподобному описанию как всей дальнейшей колебательной структуры, так и значения энергии связи. Мы осуществили такое построение для основного состояния молекулы кислорода, колебательные частоты которой рассчитаны с высокой точностью. Константы $v_{e} x_{e}$ были усреднены на интервалах $v=0-3$ и $v=0-20$. Второй вариант неплохо воспроизводит положение уровней - отклонения частот на участке примерно 3/4 глубины потенциальной ямы лежат в пределах $1-2 \mathrm{~cm}^{-1}$. В первом варианте отклонения значительно больше, главным образом, из-за упомянутой аномалии в нижней части ямы. Таким образом, можно считать, что задача Морза имеет значительно большее число решений, чем два. Добавим, что подбирая значение ангармоничности $x_{e}$, чтобы по (4) найти $D_{e}$, мы по умолчанию предполагаем, что величина $v_{e}$ в $(4)$ есть гармоническая частота, характерная для параболического потенциала (закон Гука [1], формула (20.100)). Но для реального осциллятора положение всех колебательных уровней, включая нулевой, зависит от ангармоничности, и рассматривая (2) как аппроксимационное уравнение, мы придаем величинам $v_{e}$ и $v_{e} x_{e}$ только смысл подгоночных коэффициентов. При этом, конечно, соотношение (20.84) в [1] выполняется. Отсюда следует, что варьировать $v_{e} x_{e}$ можно как за счет $x_{e}$, так и $v_{e}$, или за счет их обоих, если по условиям задачи коэффициент $x_{e}$ должен удовлетворять каким-либо дополнительным требованиям.

\section{Эмпирическая характеристика ангармоничности}

При обработке экспериментальных данных большую пользу приносит экстраполяция Берджа-Шпонер, основанная на приблизительно линейной эмпирической зависимости разностей между соседними уровнями от 


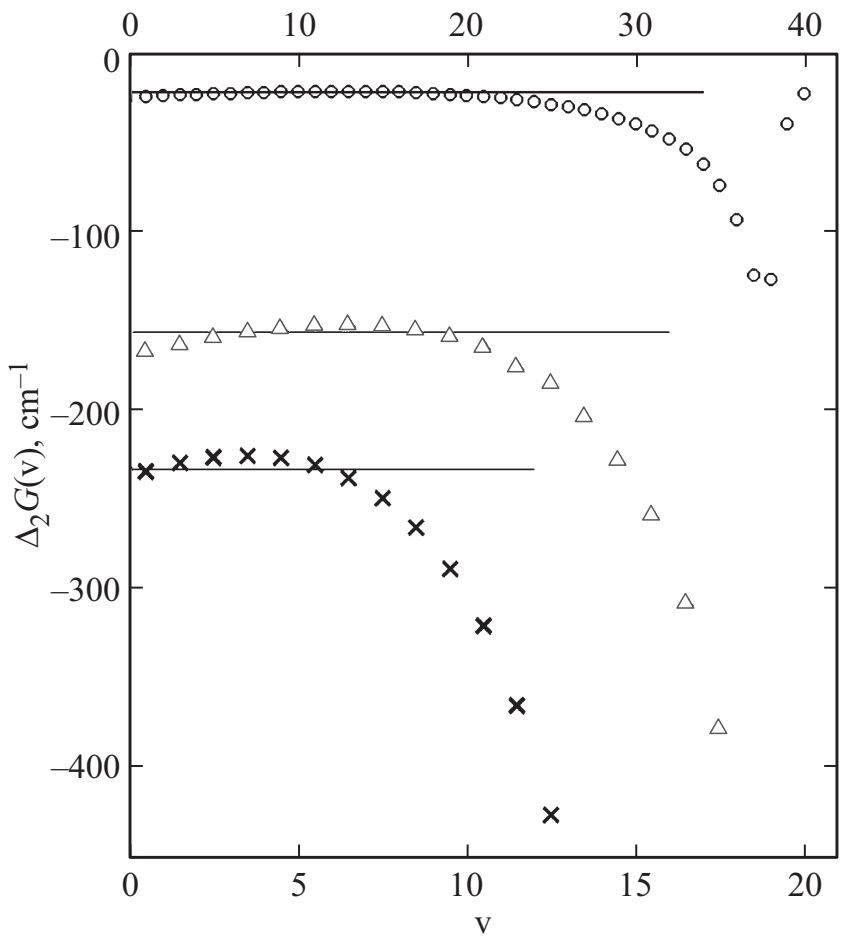

Рис. 1. Зависимости вторых разностей энергий колебательных уровней от колебательного квантового числа $F(v)=2 v_{e} x^{*}$ (уравнения (2), (7)) для молекул водорода $\mathrm{H}_{2}$ (крестики) по данным [23] и фтористого водорода НF (треугольники) [24] (нижняя ось абсцисс), а также кислорода $\mathrm{O}_{2}$ (кружки) [25] (верхняя ось абсцисс). Горизонтальные прямые проведены для каждой молекулы на уровне, соответствующем значениям постоянной ангармоничности, которые следуют из уравнения Морза: $-234.5,-157.6,-23.31 \mathrm{~cm}^{-1}$ (см. текст).

колебательного квантового числа для нижних уровней ряда двухатомных молекул $[19,20]$ :

$$
\Delta G(v+1 / 2) \equiv G(v)-G(v-1) .
$$

В координатах $\Delta G, v+1 / 2$ точки аппроксимируются прямой, экстраполяция которой до пересечения с осью абсцисс позволяет получить приближенную оценку энергии диссоциации молекулы ([1], рис. 20.9). Более подробное рассмотрение проведено в полезной работе [21]. В этих координатах уравнение (6) для морзовского осциллятора выполняется строго, точки в координатах Берджа-Шпонер лежат на прямой, наклон которой определяется значением ангармоничности. Знак и абсолютная величина отклонений от этой прямой отражают специфику реальной молекулы, ее индивидуальность, и имеет смысл использовать вторые разности, т.е. функцию

$$
\Delta_{2} G(v) \equiv \Delta G(v)-\Delta G(v-1)=F(v)
$$

для эмпирической систематики колебательных уровней двухатомной молекулы. Мы построили такие зависимости для нескольких молекул, для которых существуют надежные значения набора колебательных частот, экспериментальных или вычисленных. В том числе мы выбрали „аномальные“ термы, испытывающие изломы из-за непересечений, которые проанализированы в работе МакКой [22], предложившей оригинальный квантово-механический подход к морзовскому анализу колебательной структуры (см. ниже). В координатах (7) морзовский осциллятор представляется набором точек, лежащих на горизонтальной прямой в отрицательной полуплоскости. Для обычных термов точки располагаются вблизи этой прямой и (почти) монотонно двигаются вниз сначала медленно, а потом с ускорением приближаясь к асимптоте, что свидетельствует об увеличении ангармоничности. На рис. 1 показаны примеры зависимости (7) для основного электронного терма молекул $\mathrm{H}_{2}$, $\mathrm{HF}$ и $\mathrm{O}_{2}$. Значения ангармоничности $v_{e} x_{e}$ были вычислены так, чтобы условие Морза удовлетворительно исполнялось на центральной части терма. Для кислорода, например, как было упомянуто выше, усреднение реальных значений проводилось на интервале $v=0-20$. Горизонтальные прямые линии показывают значение $2 v_{e} x$, которое описывает положение колебательных уровней аппроксимированного морзовского осциллятора, точки не приведены.

В некоторой области вблизи асимптоты понятие ангармоничности теряет смысл из-за доминирования вандер-ваальсова взаимодействия между атомами, и мы не будем его обсуждать, а первые уровни иногда ведут себя неожиданно. Для основного терма водорода точки в интервале $v=1-5$ двигаются вверх, т.е. ангармоничность уменьшается, а затем происходит ускоренное увеличение ангармоничности вплоть до асимптоты. Эта аномалия почти не заметна в координатах Берджа-Шпонер, но Гейдон мельком упомянул о ней в книге [20] (с. 108), и, возможно, так проявляется отклонение реальной потенциальной кривой от потенциала Морза, отмеченное пунктиром в нижней части кривой на рис. 48 в книге Герцберга [7] на с. 76. Зависимость $F(v)$ для молекулы кислорода почти нормальна, хотя слабо выраженная аномалия этого типа на участке $v=1-15$ не вызывает сомнений. Наконец, для молекул $\mathrm{HF}$ и DF эта аномалия значительна, но расположена в средней части потенциала. Подробнее на этих молекулах остановимся ниже. В общем, для нормальных электронных термов зависимость $F(v)$ количественно отражает отклонение колебательных уровней реального терма от его морзовской аппроксимации, которая показана на схеме горизонтальной линией. Также на рис. 1 можно видеть интервал $\Delta v$, на котором проведено усреднение для определения исходного значения $2 v_{e} x$. Кроме того, для термов с изломом на рис. 1 удается видеть участок, в котором происходит скачкообразное изменение ангармоничности. В более сложных случаях, описанных МакКой в [22] (термы $E^{1} \Sigma_{g}^{+} \mathrm{Li}_{2}$ и $X^{1} \Sigma_{g}^{+} \mathrm{Be}_{2}$ ), сильное взаимодействие в области пересечения диабатических термов проявляется в резком скачке константы ангармоничности резонансного типа, и при этом можно усмотреть некоторый параллелизм с ее данными. Мы считаем, что 


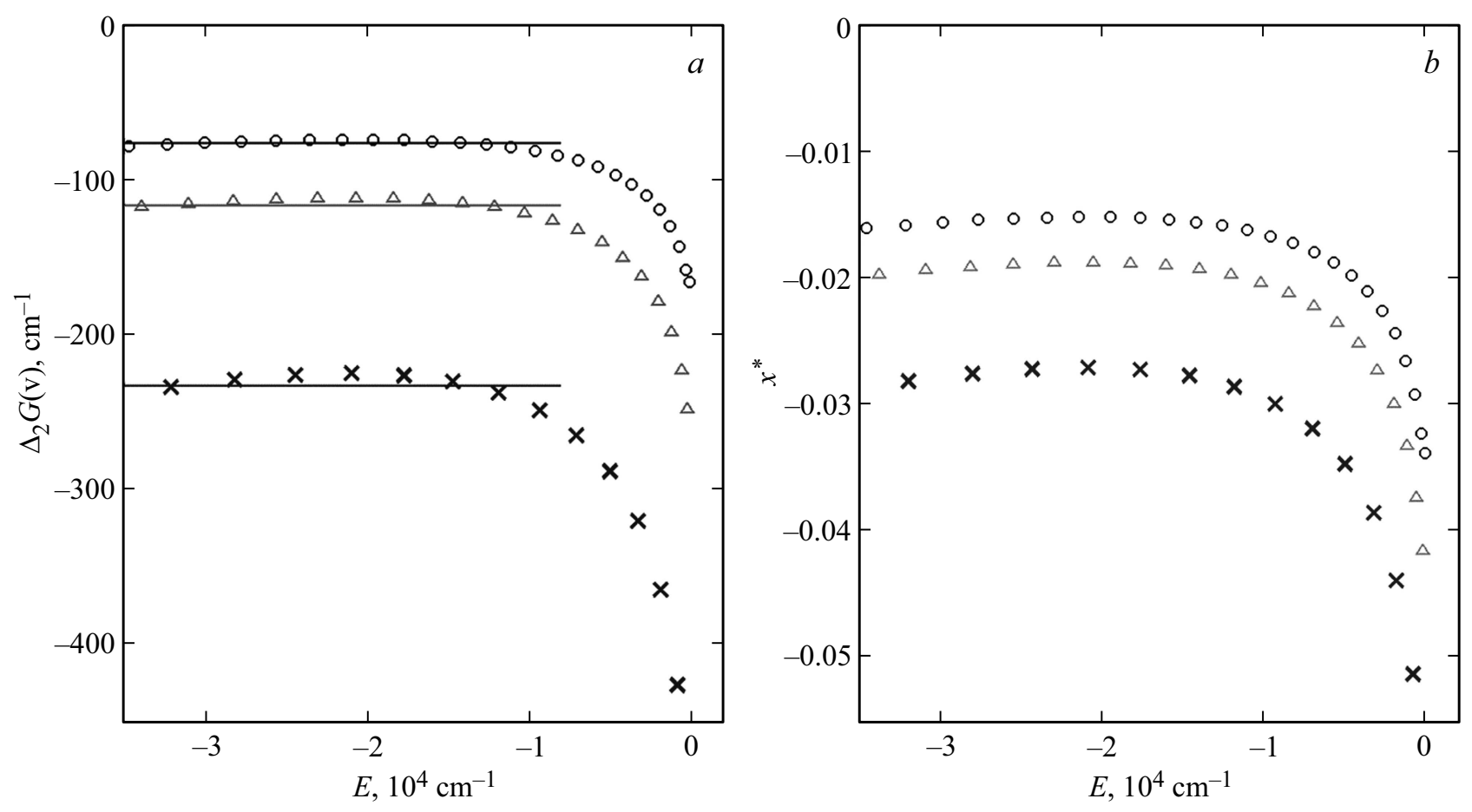

Рис. 2. Сравнение зависимостей вторых разностей $F(v)(a)$ и приведенного ангармонизма $x^{*}(E)(b)$ от энергии колебательных уровней $E$ для колебательных частот молекул $\mathrm{H}_{2}$ (крестики), $\mathrm{D}_{2}$ (треугольники), $\mathrm{T}_{2}$ (кружки) по данным [23]. Величины $\Delta_{2} G(v) \equiv G(v)-\Delta G(v-1)=2 v_{e} x^{*}(v)$ и $x^{*}(v)=2 v_{e} x^{*}(v) / 2 v_{e}$ вычислены по формуле (7).

анализ вторых разностей $F(v)$, возможно, в сочетании с подходом МакКой мог бы позволить сформулировать полезные диагностические признаки формы электронных термов простых молекул.

\section{Универсальная безразмерная шкала ангармоничности}

При накоплении таких данных возникает потребность в их обобщении, при котором величина ангармоничности была бы универсальной, не зависящей от частоты колебаний. То есть возникает потребность проанализировать возможность использования безразмерного параметра $x^{*}$ как дескриптора формы потенциала. Если ввести энергию колебательных уровней $E$ в форме $E=G(v)-D_{e}$, приняв за ноль асимптоту, то зависимость $x^{*}(E)$ (ее экспериментальную реализацию отражает функция $F(v)$ ), характеризующая отклонения ангармоничности от постоянного морзовского значения, возможно, могла бы служить следующим приближением для качественной, а в благоприятных обстоятельствах полуколичественной характеристики особенностей вида потенциала. На первом этапе важно сравнить функции $x^{*}(E)$ по возможности для нескольких изотопных форм одной и той же молекулы, чтобы выяснить, насколько хорошо проявляется в поведении этой функции условие адиабатичности, т.е. независимость формы потенциала от приведенной массы молекулы. Наиболее полезны в этом отношении гидриды из-за значительного различия колебательных квантов трех изотопов, водорода, дейтерия и трития. Использование энергии в качестве абсциссы обеспечивает универсальность условий сравнения результатов.

Наличие точных данных в литературе дает возможность построить такие зависимости для трех изотопов молекулы водорода $\left(\mathrm{H}_{2}, \mathrm{D}_{2}, \mathrm{~T}_{2}\right)$ и фтористого водорода (HF, DF, TF), полученные данные показаны на рис. 2 и 3. На рис. 2, а приведена зависимость $F(v)$ в шкале энергии $E=G(v)-D_{e}$. Значения глубины потенциальной ямы для всех изотопных форм водорода практически в адиабатическом приближении совпадают, но значение гармонической частоты для них в этом ряду уменьшается от $4401.21 \mathrm{~cm}^{-1}\left(\mathrm{H}_{2}\right)$ до $3115.5 \mathrm{~cm}^{-1}\left(\mathrm{D}_{2}\right)$ и $2546.4 \mathrm{~cm}^{-1}\left(\mathrm{~T}_{2}\right)$ [23], поэтому кривые расположены на разной высоте. В этих координатах величина аномалии Герцберга (уменьшение ангармоничности для уровней в нижней части потенциальной ямы) с увеличением массы изотопа уменьшается. Для трех изотопов мы провели построение морзовского терма описанным выше методом, усреднив значение $x^{*}$ по первым семи уровням для водорода и в том же интервале энергий для двух тяжелых изотопов. На рис. 2, $a$ горизонтальные прямые показывают уровень, на котором находятся точки морзовского осциллятора, сами точки не показаны.

На рис. 2, $b$ изображена зависимость от энергии универсальной ангармоничности $x^{*}(E)$. Зависимость от частоты исчезла, но расстояние между кривыми неожидан- 

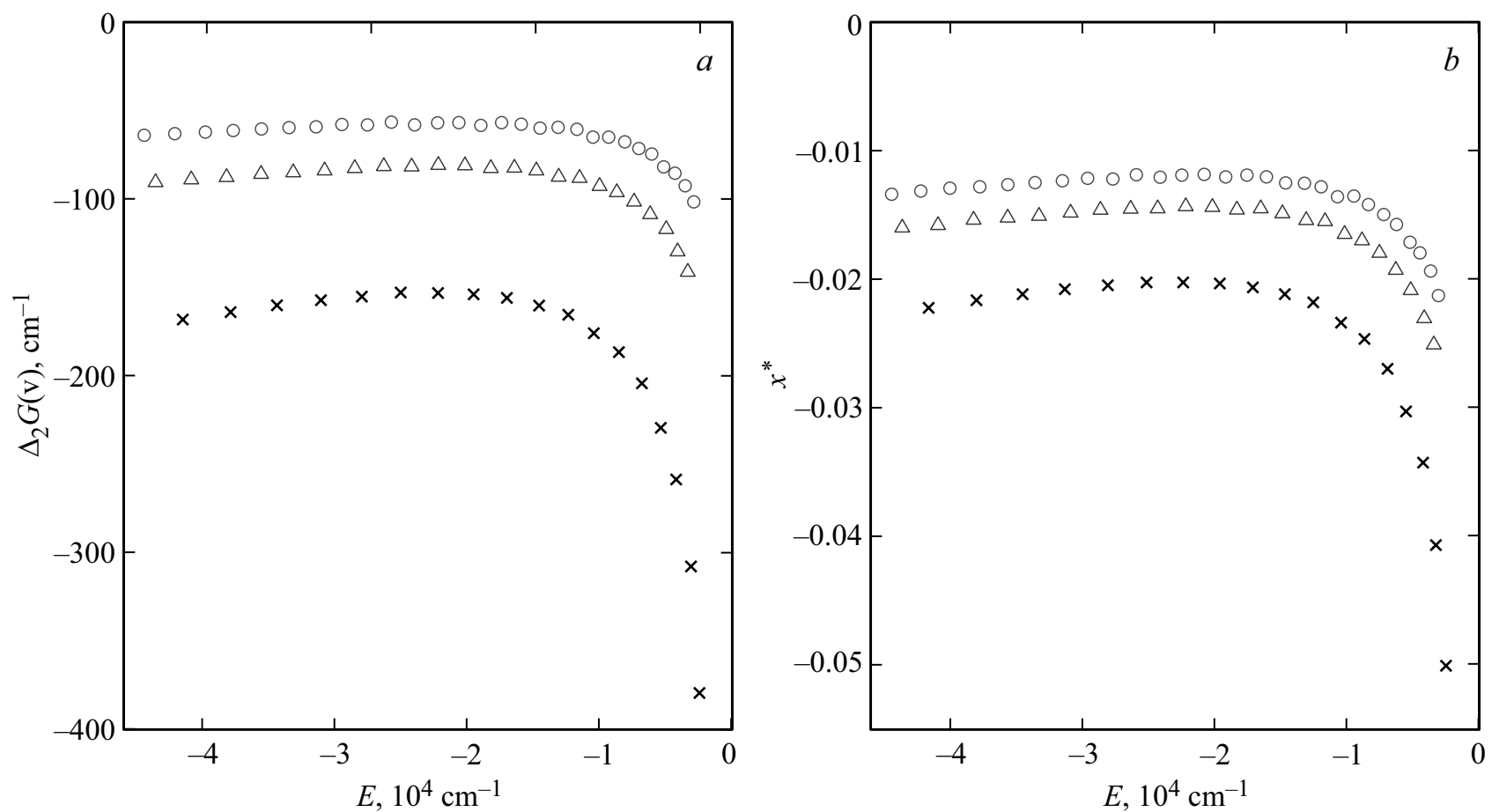

Рис. 3. Сравнение зависимостей вторых разностей $F(v)(a)$ и приведенного ангармонизма $x^{*}(E)(b)$ от энергии колебательных уровней $E$ для колебательных частот молекул HF (крестики), DF (треугольники), TF (кружки) по данным [24]). Вычисления проведены по тем же формулам, что на рис. 2.

но уменьшилось незначительно. Формально это должно означать, что при увеличении массы изотопа ангармоничность уменьшается. При этом на первый взгляд кажется, что форма кривых изменилась не одинаковым образом. Этот анализ требует специального исследования. Безразмерная универсальная ангармоничность $x^{*}$ в главной части потенциальной ямы, где она еще имеет смысл, принимает значения от 0.015 до 0.035 и для каждого изотопа меняется в интервале шириной примерно 0.015 единиц. Наивысшая ангармоничность наблюдается у терма водорода $\mathrm{H}_{2}$.

По такой же схеме обработаны литературные данные по колебательной структуре основного электронного состояния молекул $\mathrm{HF}, \mathrm{DF}, \mathrm{TF}$. Результаты представлены на рис. $3, a, b$. Картина очень похожа на изотопы водорода, описанные выше - на вторых разностях заметна небольшая аномалия Герцберга, которая воспроизводится в шкале $x^{*}(E)$, но сильнее различаются интервалы $x^{*}$ и видно несовпадение кривых для разных изотопов. Подробный анализ практических возможностей данного подхода имеет смысл проводить после накопления экспериментальных данных, включающих „аномальные“ электронные адиабатические термы - именно они покажут, насколько чувствителен параметр $x^{*}$ к „особенностям“ таких термов.

В заключение сравним зависимость вторых разностей $F(v)$ от массы изотопов. Из (7) следует, что они должны быть пропорциональны приведенным массам молекул (оба сомножителя в $v_{e} x^{*}$ зависят от корня из приве- денных масс). На рис. 4, $a$ отложены вторые разности для $\mathrm{D}_{2}$ и $\mathrm{T}_{2}$ (как на рис. 2, кружки и треугольники) и наложенные на них вторые разности для водорода, поделенные на отношение приведенных масс дейтерия и водорода (т.е. на 2) и трития и водорода (т.е. на 3) (крестики). Такая же операция проведена с данными для изотопов HF (рис. $4, b$ ). Совпадение прекрасное на всем интервале энергии, так что изотопный эффект не вносит искажений заметного масштаба в зависимость $x^{*}(E)$.

В частности, становится очевидным, что вторые разности в шкале волновых чисел выглядят более естественно, чем в шкале колебательных квантовых чисел, и являются предпочтительными при анализе. Особенно это важно иметь в виду при рассмотрении ,аномальных“ электронных термов, в которых специфические детали расположения возмущенных колебательных уровней могут быть заметно искажены.

\section{Заключение}

Сравнение поведения колебательных прогрессий для нескольких молекул с оценкой их параметров, полученной с применением потенциала Морза, показывает, насколько точно (или неточно) данный потенциал описывает свойства двухатомных молекул, для которых доступны экспериментальные или вычисленные данные в широком интервале частот. Есть примеры, когда при удачном выборе исходного значения параметра ангармоничности удается описать колебательную структуру с 

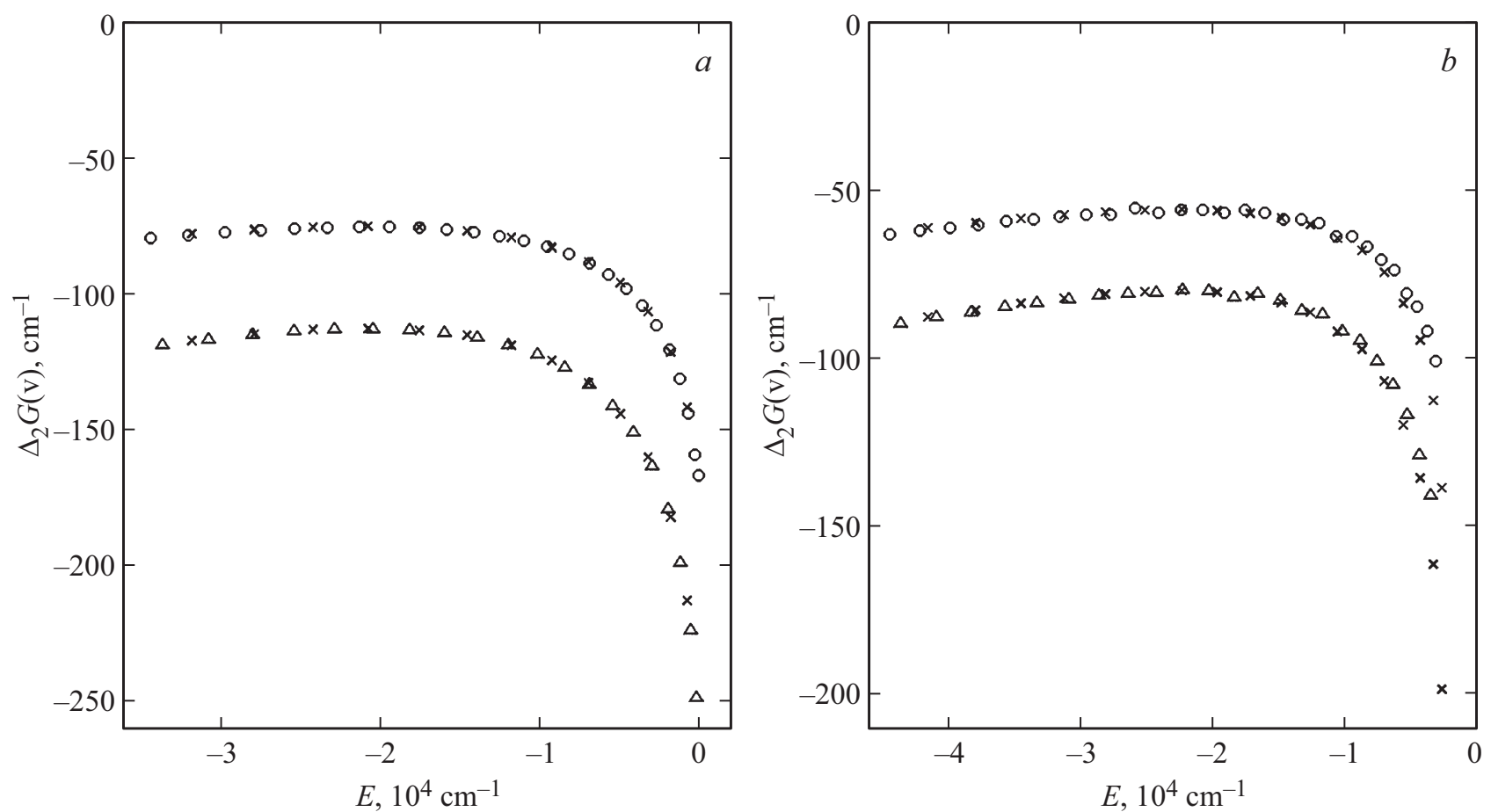

Рис. 4. Вторые разности для изотопно-замещенных молекул пропорциональны приведенным массам: $(a)$ вторые разности для $\mathrm{D}_{2}$ и $\mathrm{T}_{2}$ (как на рис. 2) и наложенные на них вторые разности для $\mathrm{H}_{2}$ (крестики), поделенные на отношение приведенных масс дейтерия и водорода $\left(M_{\mathrm{D} 2} / M_{\mathrm{H} 2}=2\right)$ и трития и водорода $\left(M_{\mathrm{T} 2} / M_{\mathrm{H} 2}=3\right) ;(b)$ вторые разности для $\mathrm{DF}$ и TF (как на рис. 3) и наложенные на них вторые разности для $\mathrm{HF}$, поделенные на отношение приведенных масс молекул $\mathrm{DF}$ и $\mathrm{HF}\left(M_{\mathrm{DF}} / M_{\mathrm{HF}}=1.905\right)$ и $\mathrm{TF}$ и $\mathrm{HF}\left(M_{\mathrm{TF}} / M_{\mathrm{HF}}=2.727\right)$.

хорошей точностью. Это означает, что для ряда молекул расположение колебательных уровней в средней части электронного терма и, менее уверенно, в самой его нижней части удовлетворяет условиям Морза с практической приемлемостью. Отклонения от постоянного ангармонизма в относительных единицах практически совпадают для изотопно замещенных молекул, что подтверждает физичность представления вторых разностей $F(v)$ как функции энергии, а не колебательного квантового числа. Безразмерная характеристика ангармонизма $x^{*}(v)$ позволяет сравнивать, по крайней мере на качественном уровне, степень ангармонизма для разных молекул. Например, для водорода $\mathrm{H}_{2}$ ангармонизм выражен сильнее, чем для HF как в среднем (усредненный постоянный ангармонизм по Морзу), так и в амплитуде отклонений от среднего.

Примечание Г.С. Денисова. Эта тема возникла во время нашей беседы с М.О. Буланиным около 15 лет тому назад, в которой мы обсуждали возникшую у нас обоих потребность найти для лекции студентам убедительные примеры практического применения функции Морза с оценкой систематических ошибок, заложенных в нее основателем. Постепенно накапливались отдельные фрагменты, и вот то, что получилось в конце концов. В этом аспекте ситуация с тех пор мало изменилась, хотя мы приводим ссылки главным образом на публикации текущего века. Мы убеждены, что результат
Михаил Олегович оценил бы по достоинству. Подробное изложение первой части данного материала принято к печати в Spectr. Acta A. 2021.

\section{Благодарности}

Авторы выражают искреннюю благодарность В.П. Булычеву и И.Г. Денисову за постоянный интерес, активные дискуссии и ценные советы.

\section{Конфликт интересов}

Авторы заявляют, что у них нет конфликта интересов.

\section{Список литературы}

[1] Ельямевич М.А. Молекулярная спектроскопия. КОМКнига, 2007. $528 \mathrm{c}$.

[2] Piela L. Ideas of Quantum Chemistry. 3-d Ed. 2020. P. 224-229.

[3] Dogra S.K., Randhava H.S. Molecular Spectroscopy. McGraw-Hill, 2012. 944 p.

[4] Svanberg S. Atomic and Molecular Spectroscopy. Springer Verlag, 4-th Ed. 2004. 588 p.

[5] McHale J.L. Molecular Spectroscopy. 2-d Ed. CRC Press, 2017. $457 \mathrm{p}$.

[6] Demtröder W. Molecular Physics. WILEY-VCH, 2005. 470 p. 
[7] Герцберг Г. Спектры и строение двухатомных молекул. М.: ИЛ, 1949. 403 c.; Herzberg G. Molecular Spectra and Molecular Structure I. Diatomic Molecules. New York., 1939.

[8] Le Roy R.J. Determining Equilibrium Structures and Potential Energy Functions for Diatomic Molecules, Ch. 6, in: Equilibrium Molecular Structures, Eds: Demaison J., Boggs J.E., Csaszar A.G. CRC Press, 2011. P. 159-204.

[9] Hua W. // Phys. Rev. A. 1990. V. 42. N 5. P. 2524.

[10] Leonard A., Deffner S. // Chem. Phys. 2015. V. 446. P. 18. doi.org/10.1016/j.chemphys.2014.10.020

[11] Walton J.R., Rivera-Rivera L.A. // J. Phys. Chem. A. 2016. V. 120. N 42. P. 8347. doi 10.1021/acs.jpca.6b05371

[12] Tuttle W.D., Harris J.P., Zheng Y., Breckenridge W.H., Wright T.G. // J. Phys. Chem. A. 2018. V. 122. N 38. P. 7679. doi 10.1021/acs.jpca.8b07139

[13] Morse P.M. // Phys. Rev. 1929. V. 34. N 1. P. 57.

[14] Krasnoshchekov S.V., Chang X. // Int. Rev. Phys. Chem. 2019. V. 38. P. 63. doi.org/10.1018/0144235X.2019.1593583

[15] Li A.Z., Harter W.G. // Chem. Phys. Lett. 2015. V. 633. P. 208. doi 10.1016/j.cplett.2015.05.035

[16] Belfakir A., Hassouni Y., Curado E.L.F. // Phys. Lett. A. 2020. V. 384. N 22. P. 126553. doi.org/10.1016/j.physleta.2020.126553

[17] Singh K.P., Kenfack A., Rost J.M., Pfeifer T. // Phys. Rev. A. 2018. V. 97. N 3. P. 33406. doi 10.1103/PhysRevA.97.03.033406

[18] Bader P., Blanes S., Kopylov N. // J. Chem. Phys. 2018. V. 148. N 24. P. 244109/7. doi.org/10.1063/1.5036838

[19] Birge R.T., Sponer H. // Phys. Rev. 1926. V. 28. N 2. P. 259.

[20] Гейдон А. Энергии диссоциации и спектры двухатомных молекул. М.: ИЛ, 1949. 302 с.; Gaydon G. Dissociation Energies and Spectra of Diatomic Molecules. Chapman \& Hall, L. 1968.

[21] Lessinger L. // J. Chem. Educ. 1994. V. 71. N 5. P. 388.

[22] McCoy A.B. // Chem. Phys. Lett. 2011. V. 501. P. 603. doi 10.1016/j.cplett.2010.11.065

[23] Wolniewicz L. // J. Chem. Phys. 1993. V. 99. N 3. P. 1851. doi.org/10.1063/1.465303

[24] Cardoen W., Gdanitz R.J. // J. Chem. Phys. 2005. V. 123. N 2. P. 024304. doi.org/10.1063/1.1949194

[25] Bytautas L., Matsunaga N., Ruedenberg K. // J. Chem. Phys. 2010. V. 132. N 7. P. 074307. doi.org/10.1063/1.3298376 